\title{
Adenovirus infections
}

\author{
Marguerite S. Pereira \\ Virus Reference Laboratory, Central Public Health Laboratory, \\ Colindale Avenue, London NW9 5 HT
}

\section{Summary}

Ninety-three adenoviruses were found in the MRC/ PHLS survey of patients in general practice and eighty-eight in those admitted to hospital, types 1, 2, 3,5 and 7 being the most frequent ones isolated. The adenoviruses accounted for $11 \%$ and $12 \%$ of all viruses and were present in $2 \%$ and $4 \%$ of patients examined in these surveys. These agents were present at all times with slightly increased frequency in the first part of each year. Fever, pharyngitis and tonsillitis were the most frequent symptoms encountered.

The adenoviruses were first described in 1953 by Rowe and his colleagues who grew what are now known as the endemic types from tonsils and adenoids removed from children at operation. Hilleman \& Werner described in 1954 some of what are known as the epidemic types, isolated from military recruits suffering from acute respiratory disease.

Antibodies to the endemic types are acquired early in life and most children have experienced infection with several different adenoviruses by the age of 5. Not much is known of these infections, many of which are probably symptomless, but there is evidence from several studies that these infections account for a proportion of the frequent respiratory illnesses of childhood (Jordan, Badger \& Dingle, 1954; Brandt et al., 1969). In the latter study approximately $7 \%$ of the cases of paediatric respiratory disease were aetiologically associated with adenoviruses. In Britain, a study of the aetiology of acute respiratory infections (Report, 1965) showed that approximately $5 \%$ of the respiratory illnesses yielded an adenovirus, a result of the same order as that obtained in the surveys now reported.

\section{Results}

\section{Isolation rate by age and type}

The results for each survey are shown in Figs. 1 and 2 . In the general practitioner survey adenoviruses were isolated from $2 \%$ of the total specimens tested and formed $11 \%$ of the total viruses isolated. The endemic types 1 and 2 predominated in all age groups, but the epidemic types 3 and 7 were more

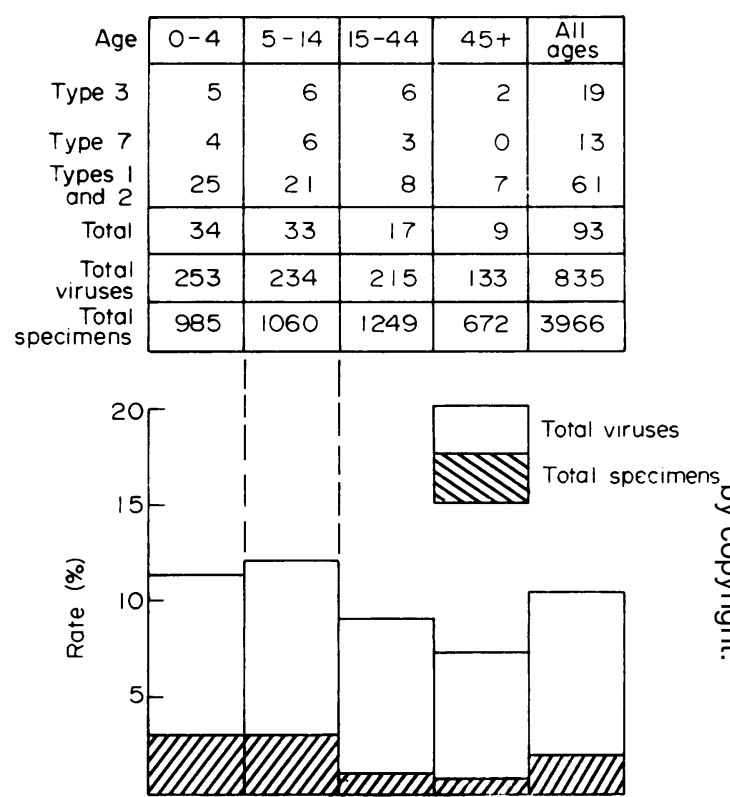

FIG. 1. Isolation rate of adenoviruses by age and type. G.P. survey. Number isolated are given in the table.

frequently isolated from older children and adults. In the hospital surveys a similar picture was found with adenoviruses isolated from $4 \%$ of the total specimens tested, forming $12 \%$ of the total viruses isolated. The endemic types were found in all age groups even in infants under 6 months. The significance of the isolation of these adenoviruses was estimated against the number of strains isolated from a control group of patients. The results are shown in Table 1 and indicate a statistically significant difference.

TABle 1. Adenoviruses

\begin{tabular}{lcc}
\hline & $\begin{array}{c}\text { Isolations } \\
\text { from } \\
\text { controls }\end{array}$ & $\begin{array}{c}\text { Isolations } \\
\text { from } \\
\text { patients }\end{array}$ \\
\hline No. of specimens tested & 280 & 648 \\
No. of adenoviruses isolated & 4 & 27 \\
Rate & 1.4 & $4 \cdot 2$ \\
\hline
\end{tabular}

Statistically significant difference $(P<0.05)$. 


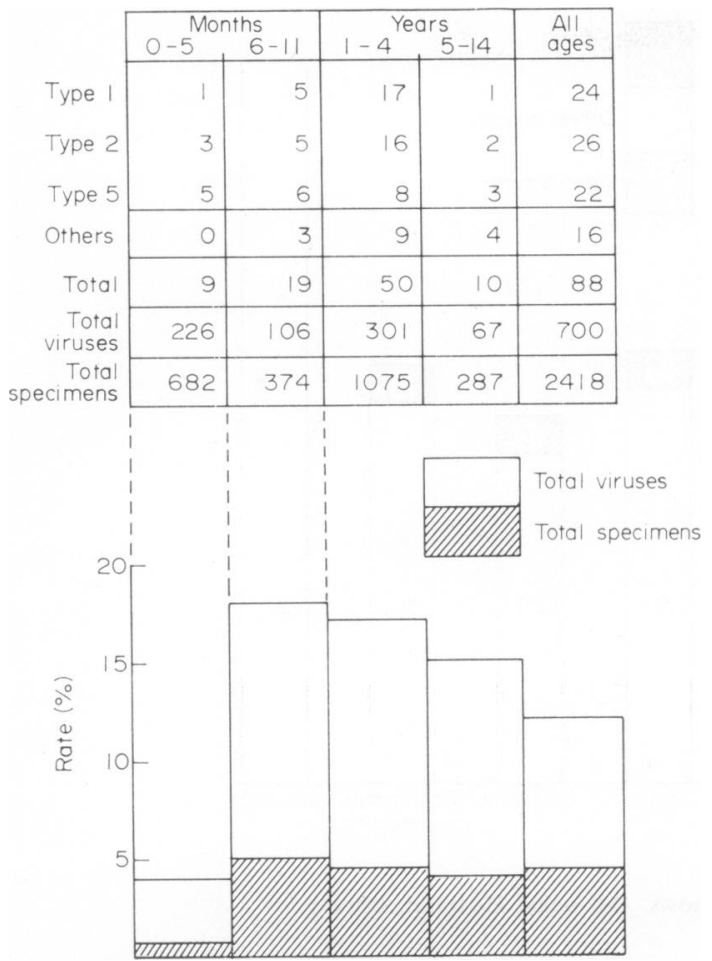

FIG. 2. Isolation rate of adenoviruses by age and type. Hospital survey. Number isolated are given in the table.

\section{Seasonal distribution}

The endemic types of adenoviruses were encountered throughout the whole period in both surveys (see Poole \& Tobin, Figs. 1 and 2, this symposium). No clear cut differences between seasons were demonstrated although there was a slight tendency towards an increase in isolations during the first half of the year.

Adenovirus type 3 was more prevalent during the winter of 1964-65 than in the second winter of the survey. In some practices there were occasional short periods where several isolations of the same serotype were made.

\section{Isolation rates of adenoviruses by diagnosis}

The findings are shown in Figs. 3 and 4. In the general practice survey adenoviruses were found associated with illnesses involving both the upper and lower respiratory tract. An adenovirus was isolated in $4 \%$ of cases of pneumonia and bronchiolitis and in $3 \%$ of common cold, pharyngitis and bronchitis and in $1 \%$ of cases of laryngitis, croup, tracheitis and influenza. No adenoviruses were isolated from cases of otitis media.

The pattern was somewhat different in the children admitted to hospital, and curiously enough the only clinical conditions yielding adenoviruses were pharyngitis and tonsillitis ( $8 \%$ ), otitis media (7\%) and common cold (3\%).

None were found in cases involving the lower respiratory tract.

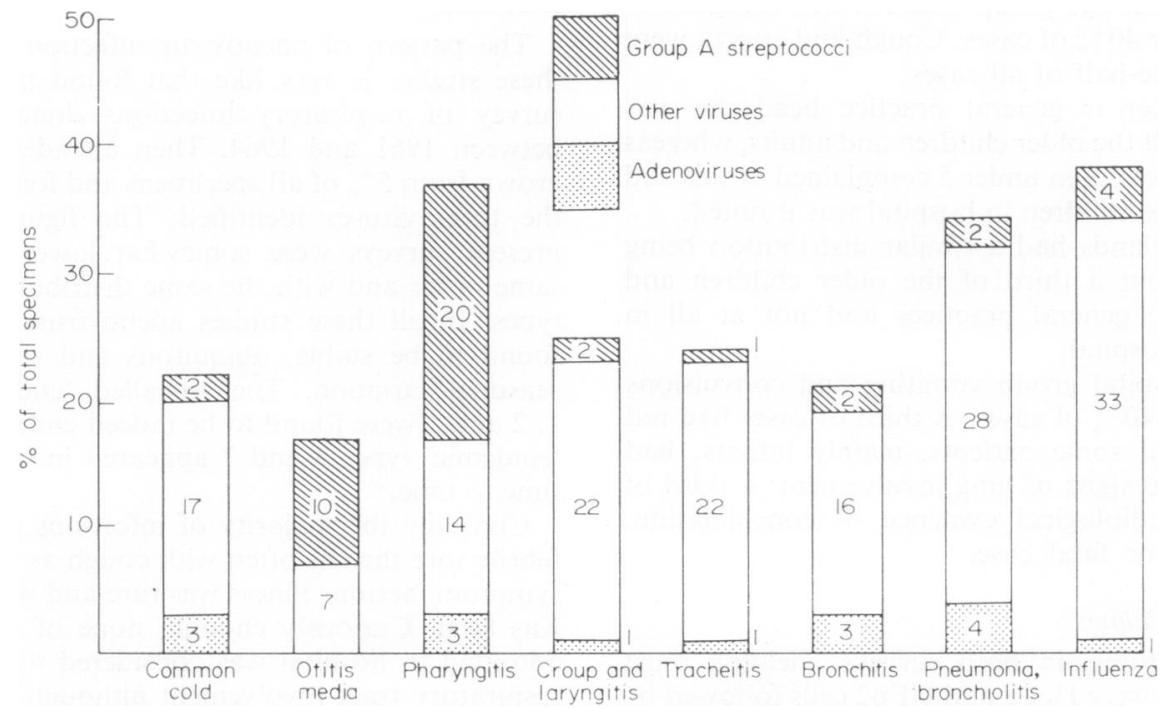

FIG. 3. Adenovirus isolations by diagnosis. All ages. G.P. survey. 


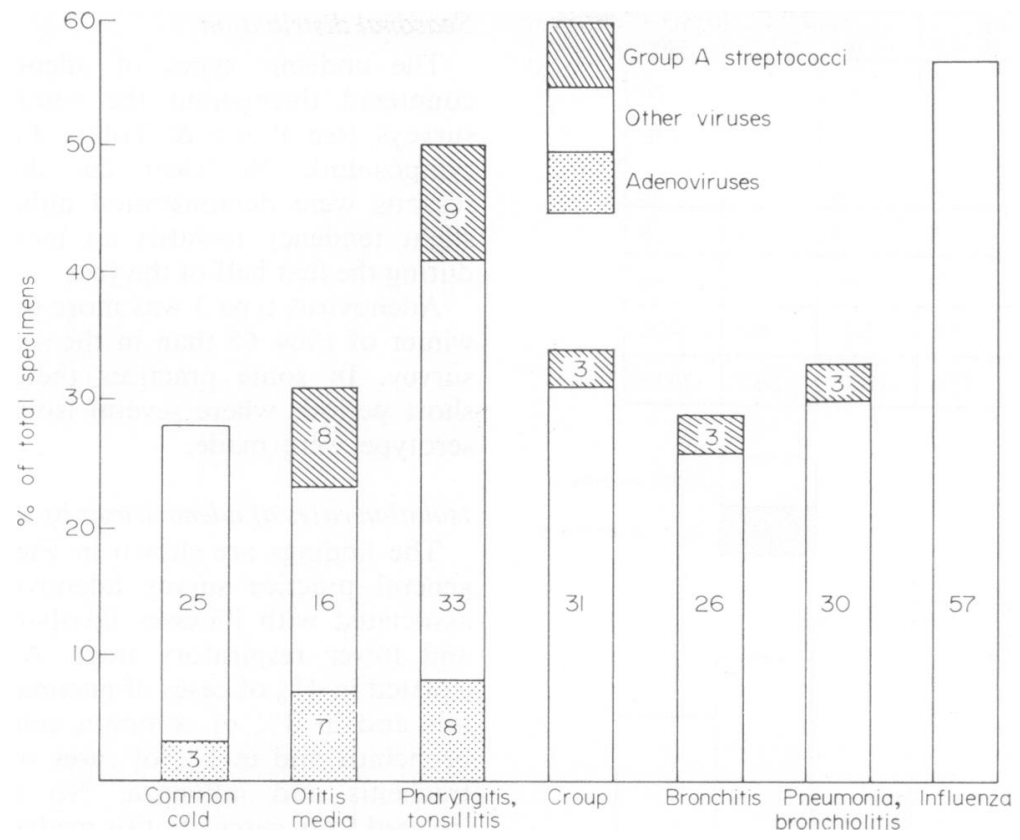

Fig. 4. Adenovirus isolations by diagnosis. All ages. Hospital survey.

\section{Clinical features}

In both surveys over $80 \%$ of adenovirus infections were accompanied by fever. This was the most frequent clinical finding (Figs. 5 and 6). The next commonest features were pharyngitis and tonsillitis which presented in two-thirds of all cases, except in the youngest age group where it was recorded in only just over $40 \%$ of cases. Cough and coryza were present in one-half of all cases.

In cases seen in general practice headache was present in half the older children and adults, whereas only $14 \%$ of children under 5 complained of this and in none of the children in hospital was it noted.

Enlarged glands had a similar distribution being noted in about a third of the older children and adults in the general practices and not at all in children in hospital.

In the hospital group vomiting and convulsions were seen in $40 \%$ of cases. A third of cases had red eardrums and some patients, mainly infants, had dyspnoea and signs of lung involvement; a third of these had radiological evidence of consolidation. There was one fatal case.

\section{Laboratory findings}

Tissue cultures, in both surveys, yielding most adenoviruses were HeLa and HEp2 cells followed by human amnion, monkey kidney, and human embryo kidney. The uneven supply of the latter did not allow confirmation of its superiority as found by other workers (Brandt et al., 1969). The virus was isolated equally from swabs taken during any of the first 5 days of illness.

\section{Discussion}

The pattern of adenovirus infection reported in these studies is very like that found in the earlier survey of respiratory infections done in Britain between 1961 and 1964. Then an adenovirus was grown from $5 \%$ of all specimens and formed $15 \%$ of the total viruses identified. The figures in these present surveys were somewhat lower but of the same order and with the same distribution of serotypes. In all these studies adenoviruses have been found to be stable, ubiquitous and showing little seasonal variation. The so-called 'endemic' types 1,2 and 5 were found to be indeed endemic and the 'epidemic' types 3 and 7 appeared in clusters from time to time.

Clinically the majority of infections presented as febrile sore throats often with cough as a prominent symptom. Serious illness was rare and only one case was fatal. Curiously enough, none of the children admitted to hospital was considered to have lower respiratory tract involvement although radiological evidence showed that some, infants particularly, had 


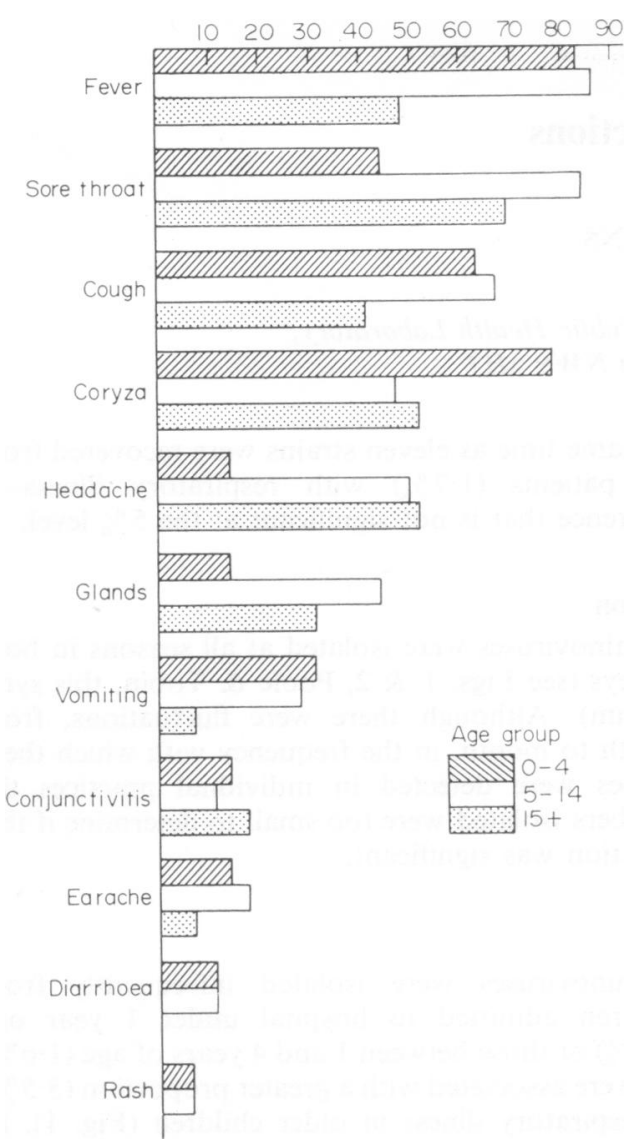

Fig. 5. Clinical features $(\%)$ of adenovirus infections All serotypes. G.P. survey.

lung changes. The high incidence of convulsions and vomiting in the hospitalized children perhaps indicates the reasons why they were admitted to hospital.

The significance of the isolation of adenoviruses in relation to disease has often been questioned.

It is known that these viruses are excreted over long periods in faeces so their presence there cannot be linked to clinical disease. When they are isolated from throats, however, it seems more probable that they may be the cause of symptoms of disease present at the time and the consistent clinical pattern described in many studies would support this association. In the 1961-64 study both endemic and epidemic adenovirus serotypes were apparently associated with disease although the endemic types were actually isolated from contacts more frequently than were the epidemic types, and in the present study sufficient controls have been examined to give clear evidence that an adenovirus in the throat is associated with clinical illness in a significant proportion of cases.

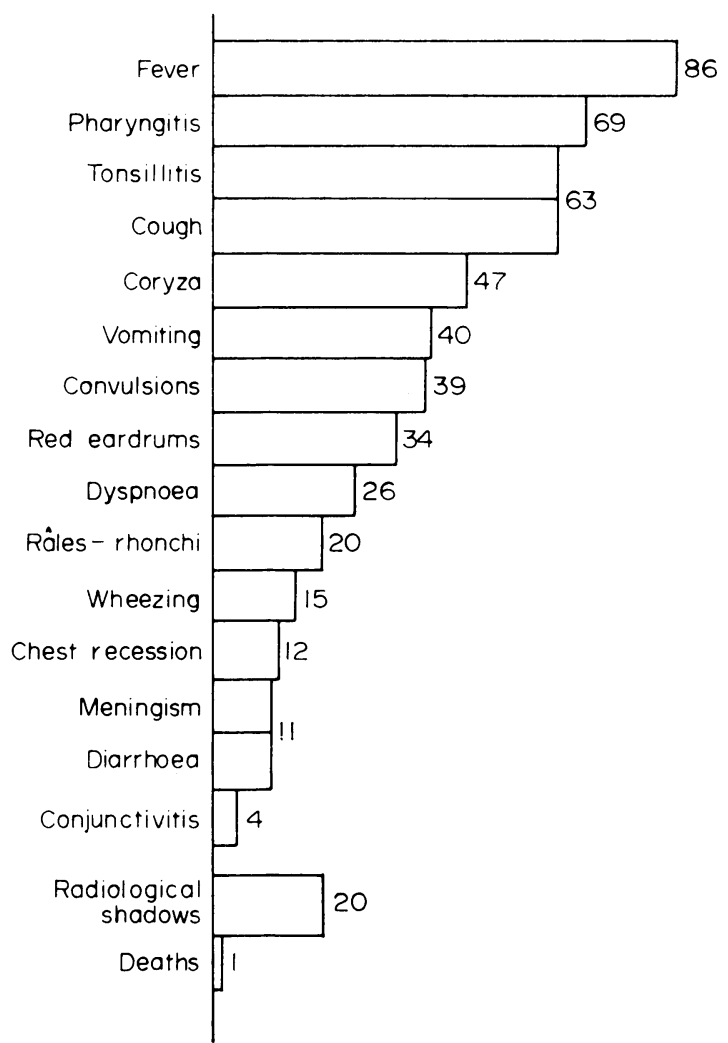

FIG. 6. Clinical features ( $\%$ ) of adenovirus infections. All serotypes. Hospital survey.

\section{References}

Brandt, C.D., Kim, H.W., Vargosko, A.J., Jeffries, B.C., Arrobio, J.O., Rindge, B., Parrott, R.H. \& Chanock, R.M. (1969) Infections in 18,000 infants and children in a controlled study of respiratory tract disease. I. Adenovirus pathogenicity in relation to serologic type and illness syndrome. American Journal of Epidemiology, 90, 484.

Hilleman, M.R. \& Werner, J.H. (1954) Recovery of new agent from patients with acute respiratory illness. Proceedings of the Society for Experimental Biology and Medicine, 85, 183.

Jordan, W.S., Badger, G.F. \& Dingle, J.H. (1958) A study of illness in a group of Cleveland families. New England Journal of Medicine, 258, 1041.

Report of the Medical Research Council Working PARTY ON ACUTE RESPIRATORY VIRUS INFECTIONS (1965) A collaborative study of the aetiology of acute respiratory infections in Britain 1961-4. British Medical Journal, 2, 319.

Rowe, W.P., Huebner, R.J., Gilmore, L.K., Parrott, R.H. \& WARD, T.G. (1953) Isolation of a cytopathogenic agent from human adenoids undergoing spontaneous degeneration in tissue culture. Proceedings of the Society for Experimental Biology and Medicine, 84, 570. 